\title{
Refresher Course on Water: Its Fascinating Science and Diverse Implications in the Natural World
}

\section{6-29 November 2015}

\author{
Sponsored by Indian Academy of Sciences, Bengaluru \\ Indian National Science Academy, New Delhi \\ The National Academy of Sciences, India, Allahabad \\ In collaboration with Field Marshal K.M. Cariappa College \\ Madikeri, Kodagu District, Karanataka
}

A Refresher Course on Water: Its fascinating science and diverse implications in the natural world, for College/ University teachers, students and interested scientists will be held at Field Marshal K.M. Cariappa College, Madikeri, Kodagu district, Karnataka from 16th November to 29th November 2015.

Water is a central ingredient of the natural world on earth, and plays an essential role as the medium in which life exists, and as a major constituent of the planet's surface, plays a central role in many processes and cycles in the natural world around us. An understanding of water in its many dimensions, from its microscopic physics and chemistry, to its roles in biological, atmospheric, environmental contexts, and as a centrally important natural resource, is thus both fascinating and an essential element of the scientific understanding of our natural world. These diverse aspects of water will be explored in this refresher course, and with water as the unifying theme, expose participants to many important aspects of contemporary science in a coherent and integrative fashion.

Topics: Basic science of water (physical, chemical and biological aspects), Water in clouds and the atmosphere, Ocean Physics, Hydrology and Water Resources, The cryosphere and climate change, surface science of water, clean water: Issues and nanotechnology solutions, water management and harvesting, water in the solar system and space.

Lecturers: Roddam Narasimha (JNCASR), Srikanth Sastry (JNCASR), Debasis Sengupta (IISc), Pradeep Mujumdar (IISc), R. Shankar (IMSc), Nimesh Patel (CfA), T. Pradeep (IITM), S Vishwanath (BES).

College/university teachers/students/research scholars with at least a Master's degree in science or degree in engineering area can apply. Teachers/Research Scholars who wish to participate in this Refresher Course should submit their completed application form online by clicking on the following link:

http://web-japps.ias.ac.in:8080/Refreshcourse/RCWS.jsp

A hard copy of the application through the Head of the institution should also be sent by speed post to:. The applications should be sent by post or email to:

Prof. Srikanth Sastry, c/o Dr T D Mahabaleswara , Coordinator (Lecture Workshops \& Refresher Courses) Science Education Panel, Indian Academy of Sciences, C.V. Raman Avenue, Sadashivanagar, Post Box No. 8005 Bangalore 560 080, Email: sep@ias.ernet.in (CC: to sastry@jncasr.ac.in and jagannathnettar@yahoo.co.in)

Selected teachers will be provided local hospitality and round trip shortest train fare (3-tier AC).

Last date for receipt of applications: 30 September, 2015 